\title{
Assistência odontológica ao paciente oncológico pós-terapia antineoplásica
}

\author{
Dental care to the oncological patient after antineoplastic therapy \\ Atención dental al paciente oncológico después de la terapia antineoplástica
}

Recebido: 02/05/2021 | Revisado: 09/05/2021 | Aceito: 13/05/2021 | Publicado: 31/05/2021

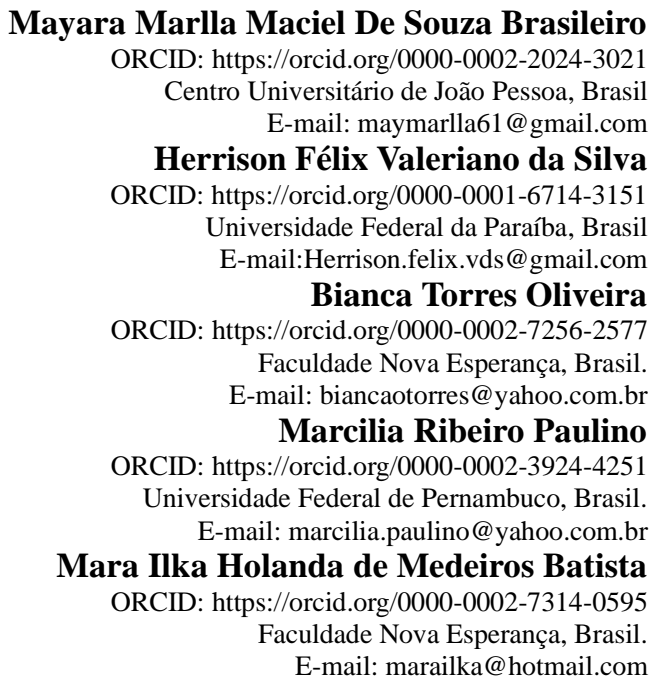

\begin{abstract}
Resumo
Este trabalho teve como objetivo apresentar os cuidados odontológicos para as manifestações bucais que podem ocorrer depois do tratamento antineoplásico. Como metodologia, foi realizada busca nas bases de dados Pubmed, Lilacs e Bibliografia Brasileira de Odontologia (BBO), além de capítulos de livros, e dissertações. Baseados nos estudos analisados foram encontradas as alterações bucais mais frequentes e propôs algumas formas de tratamento odontológico para o suporte a estes indivíduos, os quais receberam terapia antineoplásica, com o objetivo de proporcionar aos profissionais da saúde opções terapêuticas que melhorem a qualidade de vida dos indivíduos com câncer, após a análise dos artigos selecionados, concluímos que a participação dos profissionais de odontologia na terapia integral do câncer é indispensável, o cirurgião-dentista deve estar capacitado para o atendimento ao paciente oncológico, ao recebê-lo antes do início das terapias, deverá realizar uma avaliação clínica e radiográfica da sua boca para identificar e tratar todo e qualquer foco de infecção nessa fase.
\end{abstract}

Palavras-chave: Quimioterapia; Radioterapia; Câncer; Cirurgião-dentista; Neoplasia.

\begin{abstract}
This study aimed to present dental care for oral manifestations that may occur after antineoplastic treatment. As a methodology, a search was carried out in the databases Pubmed, Lilacs and Bibliography of Brazilian Dentistry (BBO), in addition to book chapters and dissertations. Based on the studies analyzed, the most frequent oral changes were found and proposed some forms of dental treatment to support these individuals, who received antineoplastic therapy, in order to provide health professionals with therapeutic options that improve the quality of life of individuals with cancer, after analyzing the selected articles, we concluded that the participation of dental professionals in comprehensive cancer therapy is essential, the dental surgeon must be trained to care for cancer patients, when receiving it before the start of therapies, you should perform a clinical and radiographic evaluation of your mouth to identify and treat any and all outbreaks of infection at this stage.
\end{abstract}

Keywords: Chemotherapy; Radiotherapy; Cancer; Dental surgeon; Neoplasia.

\section{Resumen}

Este estudio tuvo como objetivo presentar la atención odontológica de las manifestaciones bucales que pueden ocurrir después del tratamiento antineoplásico. Como metodología, se realizó una búsqueda en las bases de datos Pubmed, Lilacs y Bibliography of Brazilian Dentistry (BBO), además de capítulos de libros y disertaciones. A partir de los estudios analizados se encontraron los cambios bucales más frecuentes y se propusieron algunas formas de tratamiento odontológico para apoyar a estos individuos, que recibieron terapia antineoplásica, con el fin de brindar a los profesionales de la salud opciones terapéuticas que mejoren la calidad de vida de los individuos con cáncer. luego 
de analizar los artículos seleccionados, concluimos que la participación de los profesionales odontológicos en la terapia integral del cáncer es indispensable, el cirujano dentista debe estar capacitado para atender a los pacientes con cáncer, al recibirlo antes del inicio de las terapias, se debe realizar una evaluación clínica y radiográfica de la boca para identificar y tratar todos y cada uno de los brotes de infección en esta etapa.

Palabras clave: Quimioterapia; Radioterapia; Cáncer; Cirujano dentista; Neoplasia.

\section{Introdução}

O câncer caracteriza-se pela multiplicação exacerbada de células neoplásicas, que provocam a desorganização durante a divisão celular prejudicando seu metabolismo. O que diferencia uma célula não neoplásica de uma célula neoplásica é o seu poder de crescimento. Essas células quando se espalham pelo corpo acabam provocando uma expansão, que se entende por metástase (INCA, 2011).

Nos dias atuais o câncer é uma das principais causas de morte no mundo e, causando em 2008 cerca de 7,6 milhões de mortes. Em 2030 espera-se que continue a aumentar as mortes por câncer em todo o mundo chegando a atingir o número de 13,1. Para tratar o câncer temos como métodos terapêuticos a cirurgia, radioterapia ou quimioterapia, podendo ser realizado apenas um método ou a associação de dois ou mais de acordo com as características da doença. Sendo assim o objetivo maior é curar a doença ou prolongar significativamente a sobrevivência e melhorar a qualidade de vida do paciente (OMS, 2014).

Por ser tão complexo, é de extrema importância uma equipe multiprofissional de saúde capacitada, com o objetivo de proporciona uma melhor qualidade de vida para o paciente. Faz-se necessária a existência do cirurgião dentista na equipe, dentre esses profissionais por vários motivos. O dentista atua de forma preventiva, através de um acompanhamento e orientação de higiene oral, e de forma curativa, eliminando focos de infecção presentes que podem interferir no tratamento proposto ao paciente ou tratando manifestações orais que surgem em virtude do tratamento. O dentista deve intervir propiciando uma melhora das condições orais do paciente, garantindo um maior conforto ao paciente. Isso pode ser feito de acordo com a necessidade do mesmo (Santos, 2010).

É importante que o dentista atue em conjunto com a equipe multiprofissional, sendo responsável, juntamente com o radio terapeuta pela prevenção e tratamento das possíveis complicações orais, sendo que o dentista é quem vai intervir diretamente na cavidade oral com o intuito de minimizar as alterações (Lima, 2005).

Já é comprovado que o cirurgião-dentista deve fazer parte da equipe multiprofissional no tratamento de pacientes oncológicos, principalmente na pré-terapia e na pós-terapia. Entretanto se observa na literatura uma carência de trabalhos que abordem esse tipo de tratamento, o que justifica o presente estudo.

Essa revisão tem como objetivo apresentar a importância da assistência odontológica ao paciente oncológico pósterapia antineoplásica, conhecer a atuação do cirurgião dentista como profissional importante na equipe multidisciplinar no tratamento do paciente oncológico; perceber a necessidade do acompanhamento do cirurgião-dentista no tratamento pré, trans e pós-terapia antineoplásica; e, identificar as complicações orais que ocorrem em pacientes oncológicos pós-terapia antineoplásica.

\section{Metodologia}

A sistemática de procedimento escolhida no presente trabalho foi a pesquisa bibliográfica, que se trata da busca de uma problematização de um tema de pesquisa a partir de referências publicadas, analisando e discutindo as contribuições culturais e cientificas. Ela constitui uma excelente técnica para fornecer ao pesquisador a bagagem teórica, de conhecimento, e o treinamento científico que habilitam a produção de trabalhos originais e pertinentes (Carvalho et al., 2004).

A revisão da literatura é uma parte vital do processo de investigação. Esta envolve localizar, analisar, sintetizar e interpretar a investigação prévia (revistas cientificas, livros, anais de congressos, resumos, etc.) relacionada com a sua área de 
estudo; é, então, uma análise bibliográfica pormenorizada, referente aos trabalhos já publicados sobre o tema. A revisão da literatura é indispensável não somente para definir bem o problema, mas também para obter uma ideia precisa sobre o estado atual dos conhecimentos sobre um dado tema, as suas lacunas e a contribuição da investigação para o desenvolvimento do conhecimento (Bento, 2012).

O presente trabalho teve como material de estudo livros, artigos científicos, dissertações e teses, encontrados nas bases de dados (Bireme, BBO, SCIELO, MEDLINE, LILACS) da internet e na biblioteca físicas do Centro Universitário de João Pessoa - UNIPÊ. Para seleção das fontes foram consideradas como critério de inclusão as bibliografias que abordam a atuação do cirurgião dentista na terapia antineoplásica em pacientes oncológicos.

Foram incluídos no estudo artigos, teses, dissertações, trabalhos de conclusão ou de revisão sistemática publicados entre 2000 a 2020. Foram excluídos artigos em outro idioma que não fosse português ou inglês e que não enfatizaram a relação entre o cirurgião dentista e a terapia antineoplásica a pacientes oncológicos.

Foi realizada uma leitura exploratória do material bibliográfico, que logo em seguida foi catalogado em conformidade com o tema proposto. Depois dos artigos serem sidos lidos de maneira mais seletiva, foram classificados na ordem de data de publicação e, agrupados em subtemas de acordo com a sequência linear de entendimento acerca do assunto. Os descritores utilizados no estudo foram: câncer oral, quimioterapia, radioterapia, terapia antineoplásica, oncologia e cirurgião-dentista.

\section{Resultados e Discussão}

$\mathrm{O}$ câncer é uma das doenças que mais causam medo na sociedade, por ser um estigma de mortalidade e dor. A nomenclatura científica de câncer refere-se ao termo neoplasia, especificamente aos tumores malignos, como sendo uma patologia caracterizada pelo crescimento descontrolado de células transformadas (Almeida, 2005). Associado a esses dados o estudo de Fontes e Alvim (2008) indaga que o surgimento de casos de câncer tem crescido consideravelmente em todo mundo, principalmente a partir do século XX, constituindo-se em um dos mais importantes problemas de saúde pública, tanto em países desenvolvidos, como naqueles em desenvolvimento, sendo responsável por mais de seis milhões de óbitos a cada ano no mundo.

Segundo o Inca (2001) a terapia contra o câncer pode ser executada através de cirurgia, radioterapia, quimioterapia ou por ambas. A radioterapia exclusiva pode ser indicada em pacientes considerados inoperáveis devido à grande extensão do tumor. Atentando para essas duas modalidades terapêuticas (radioterapia e quimioterapia) é sabido que as mesmas provocam danos principalmente nas células com alta taxa de divisão.

Associado ao tratamento de radioterapia, o cirurgião-dentista deve atuar nas infecções e alterações que já estejam presentes na cavidade oral, além de prevenir novas mudanças. Basicamente, antes da radioterapia, devem ser tomadas medidas como: instruções de higiene oral, profilaxia, aplicação tópica de flúor, tratamentos restauradores, tratamento periodontal, exodontias e necessária eliminação de traumas. Durante a terapia, deve-se tomar as seguintes medidas: controle e orientação de higiene bucal, uso de flúor tópico, exodontia são contraindicadas. Ao final da radioterapia, devem ser tomadas as seguintes medidas: controle e orientação de higiene bucal, evitar exodontias por um ano e devem ser feitas visitas regulares ao consultório odontológico (Salazar, 2008).

Estudos mostraram que por consequência, a mucosa oral normal torna-se alvo primário para a toxicidade em virtude de sua alta taxa de renovação celular. As complicações mais comuns observadas em pacientes irradiados são mucosite, xerostomia, dor, hipossalivação, cáries de radiação, diugesia e osteorradionecrose, que podem levar à dificuldade de alimentação, resultando em desidratação e desnutrição, essas implicações influenciam alguns pacientes a desistir do seu tratamento (Daniel et al., 2006; National Cancer Institute, 2018). 
Como consequência da má higienização bucal o surgimento de alterações estomatológicas está associado ao processo insatisfatório dos cuidados com a cavidade oral, levando, portanto, ao desenvolvimento de complicações nesse local. Além disso, a radioterapia foi a modalidade terapêutica mais empregada, sendo responsável pela maioria das disfunções supracitadas de acordo com o estudo de Paiva et al., (2010).

Corroborando com o pensamento dos autores assim citados anteriormente os elementos de risco para evolução dessas complicações orais durante e após o tratamento são a preexistência de infecções bucais, bem como a falta de cuidados adequados. Lesões cariosas, fraturas dentárias, remanescentes radiculares, cálculo dental e doença periodontal contribuem para o desenvolvimento de infecções locais e podem servir de foco para infecções sistêmicas. Dessa forma se faz necessário o acompanhamento do cirurgião-dentista para diagnosticar, orientar e tratar (Howell, 2003).

Pacientes submetidos ao tratamento oncológico podem apresentar dificuldades na higienização bucal, levando ao comprometimento das estruturas orais ou exacerbando condições muitas vezes já instaladas no indivíduo. Assim, o monitoramento da saúde bucal durante e após o término do tratamento é essencial (Antunes, 2005). Validando, portanto, essa linha de raciocínio com o pensamento dos autores Almeida et al., (2015), do modo que estes explanam da mesma opinião, onde a atenção com a saúde bucal realizada pela equipe odontológica tem como objetivo um tratamento integral do paciente, pois é através da cavidade oral que os microrganismos patogênicos adentram, causando infecções sistêmicas.

A implementação de protocolos de cuidados com a cavidade oral ameniza os riscos da instalação de doenças sistêmicas e infecções. Assim, como relatado por Aranega et al., (2012) afirmando que a equipe odontológica no âmbito hospitalar é fundamental e indispensável no que tange a assistência de qualidade ao paciente interno, bem como a um prognostico positivo, fazendo necessário à manutenção da saúde bucal, além da maior integração da odontologia com toda a equipe multidisciplinar.

Eliminação de complicações orais e implementação de protocolos concebidos para manter o máximo de saúde deve ser componentes da avaliação dos pacientes, cuidados devem ser tomados antes da RT começar, o pacientes deve fazer uma consulta abrangente com o dentista semanas antes do tratamento de radiação se iniciar. Desta forma, fornecer um adequado intervalo para a cicatrização do tecido, no caso de procedimentos, incluindo extração dentária, tratamento endodôntica e raspagens periodontais (Devi; Singh, 2014).

Ccotidianamente o combate contra o câncer vem aumentando e não pode ser diferente no consultório odontológico. Em muitos casos, o cirurgião-dentista é o primeiro profissional a suspeitar e diagnosticar o câncer de boca; no entanto poucos dentistas atuam na sua prevenção (Prado; Passarelli, 2009).

O presente trabalho buscou na revisão de literatura, a melhor forma de prevenção do câncer bucal, como o cirurgiãodentista deve sempre direcionar, discutir e incentivar o paciente quanto à cessação do ato de fumar, o consumo moderado de bebidas alcoólicas, alimentação saudável, uma higiene bucal satisfatória e problemas com a exposição solar, além de diagnosticar e tratar precocemente as lesões pré-câncer, eliminar fatores irritantes na mucosa bucal além de orientar o paciente a fazer o autoexame da boca regularmente (Kujan et al. 2006).

A indispensabilidade de uma abordagem multiprofissional resultou, à medicina e odontologia, alterações profundas onde o acompanhamento do cirurgião-dentista especializado no corpo clínico dos hospitais tem sido cada vez mais forte, uma vez que, o futuro aponta para uma tendência ainda mais acentuada da atuação deste profissional neste campo de trabalho (Morais et al., 2006).

\section{Conclusão}

Pensando na melhor opção para os pacientes em tratamento do câncer, o profissional da área odontológica deve estar habilitado para diagnosticar, prevenir, controlar e tratar as complicações orais que surgem durante as diversas fases do 
tratamento oncológico. Condutas clínicas simples como higiene bucal, controle do biofilme oral podem impedir ou melhorar as manifestações secundárias na boca provocadas pelo tratamento do câncer.

A participação dos profissionais de odontologia na terapia integral do câncer é indispensável, o cirurgião-dentista deve estar capacitado para o atendimento ao paciente oncológico. Ao recebê-lo antes do início das terapias, deverá realizar uma avaliação clínica e radiográfica da sua boca para identificar e tratar todo e qualquer foco de infecção nessa fase.

Esta revisão aponta para a necessidade de novos trabalhos sobre essa temática, afim de obter novas evidencias cientificas, sobre métodos e técnicas, que minimize cada vez mais, as complicações em pacientes em tratamento para o câncer.

\section{Referências}

Almeida, V. L. et al. (2005). Câncer e agentes antineoplásicos ciclo-celular específicos e ciclo-celular não específicos que interagem com o dna: uma introdução. Quim. Nova. 28(1), 118-129.

Almeida, R. C. C. et al. (2015). A importância do tratamento odontológico em pacientes internados em unidades de terapia intensiva. Encontro de Extensão, Docência e Iniciação Científica - EEDIC - (Resumo). Centro Universitário Católica de Quixabá. Quixabá.

Albuquerque, A. R.; Morais; L. L. V. \& Sobral, V. P. A. (2007). Protocolo de atendimento odontológico a pacientes oncológico pediátricos. RevOdontol UNESP. 36(3), 275-80.

Alves, V. T. E. et al. (2013). Aspectos relacionados ao câncer oral de interesse na periodontia. Braz J Periodontol, $23,4$.

Antunes, H. S. (2005). Low power laser therapy in the prevention of induced oral mucositis in bone marrow transplantion patients. São José dos Campos: Univap, 2005. Dissertação (Mestrado em Bioengenharia) Programa de Pós Graduação em Bioengenharia. Universidade do Vale do Paraíba.

Araújo, S. S. C.; Padilha, D. M. P. \& BaldisserO, J. (2007). Saúde Bucal e Qualidade de Vida em Pacientes com Câncer de Cabeça e Pescoço. R. Fac. Odontol. Porto Alegre. 48(1/3), 73-76.

Aranega, A. M. et al. Qual a importância da odontologia hospitalar? Revista Brasileira odontologia, 69(1), 3-90.

Bento, A.V. (2018). Como fazer uma revisão de literatura: considerações teóricas e práticas. http://www3.uma.pt/bento/Repositorio/Revisaodaliteratura.pdf.

Bonan, P.R.F. et al. (2005). Aspectos clínicos, biológicos, histopatológicos e tratamentos propostos para mucosite oral induzida por radioterapia: revisão de literatura. Revista Brasileira de Cancerologia. 51(3), 235-242.

Brasil, Ministério da saúde. (2013). Manual de bases técnicas da oncologia: sistema de informações ambulatoriais. (14a ed.), Ministério da Saúde.

Brasil. (2008). Ministério da Saúde. Instituto Nacional de Câncer (INCA). Ações de enfermagem para o controle do câncer: uma proposta de integração ensino-serviço. (3a ed.), MS/INCA.

Cardoso, M. F. A. et al. (2005). Prevenção e controle das seqüelas bucais em pacientes irradiados por tumores de cabeça e pescoço. Radiol. Bras. 38(2), 107115.

Carvalho, D. et al. (2004). Pesquisa Bibliográfica. Universidade Federal de Goiás, Faculdade de Comunicação e Biblioteconomia. Trabalho acadêmico realizado por alunos do $5^{\circ}$ período de Comunicação Social com habilitação em Jornalismo. http://www.scielo.br/pdf/eins/v8n1/pt_1679-4508-eins-8-1-0102.

Costa, P. \& Leite, R. C. B. O. (2009). Estratégias de enfrentamento utilizadas pelos pacientes oncológicos submetidos a cirurgias mutiladoras. Revista Brasileira de Cancerologia. 55(4), 355-364.

Czlusniak, G. D.\& Kroetz, F. M. (2004). Alterações bucais e condutas terapêuticas em pacientes infanto-juvenis submetidos a tratamentos anti-neoplásicos. UEPG Ci Biol Saúde. 9(2), 41-48.

Daniel, F.I. et al. (2006). Carcinoma de células escamosas em rebordo alveolar inferior: diagnóstico e tratamento odontológico de suporte. J Bras Patol Med Lab. 42(4), 279-283.

Devi, S. \& Singh, N. (2014). Dental care during and after radiotherapy in head and neck cancer. National Journal of Maxillofacial Surgery. 5(2).

Faria, M. T. (2017). Atendimento odontológico ao paciente com câncer: Orientação para cirurgiões dentistas.

Fischer, D. J. \& Epstein, J. B. (2008). Management of patients who have undergone head and neck cancer therapy. Dent Clin North Am. 52, $39-60$.

Freitas, D. A. et al. (2011). Sequelas bucais da radioterapia de cabeça e pescoço. Rev. CEFAC. 13(6), 1103-8.

Fontes, C. A. S. \& Alvim, N. A. P. (2008). A relação humana no cuidado de enfermagem junto ao cliente com câncer submetido à terapêutica antineoplásica. Acta Paul Enferm. 21, 77-83.

Ghelardi, I. R. et al. (2008). A necessidade da avaliação e tratamento odontológico préradioterapia. Prática Hospitalar. n.58.

Grimaldi, N. et al. (2005). Conduta do cirurgião dentista na prevenção e tratamento da osteorradionecrose: revisão de literatura. Revista Brasileira de Cancerologia. 51(4), 319-324. 
Goursand, D. et al. (2006). Seqüelas bucais em crianças submetidas à terapia antineoplásica: causas e definição do papel do cirurgião dentista. Arquivos em Odontologia. 42(3), 161-256.

HespanhoL, F. L. et al. (2010). Manifestações bucais em pacientes submetidos à quimioterapia. Ciênc saúde coletiva. (15), 1085-1094.

Howell, R. E.; Wright, B. A. \& Dewar, R. (2003). Trends in the incidence of oral cancer in Nova Scotia from 1983 to 1997. Oral Surg Oral Med Oral Pathol Oral Radiol Endod, 95(2), 205-12.

Instituto Nacional Do Câncer. (2001). Carcinoma epidermóide da cabeça e pescoço. Revista Brasileira Cancerologia, 47(4), 361-76.

Instituto Nacional Do Câncer José Alencar Gomes Da Silva. (2011). ABC do Câncer: abordagens básicas para o controle do câncer.

Jham, B. C. \& Freire, A. R. S. (2006). Complicações bucais da radioterapia em cabeça e pescoço. Rev Bras Otorrinolaringol. 72(5).

Kujan, O. et al. (2006). Opinion and attitudes of the UK's GDPs ans specialists in oral surgery, oral medicine and surgical dentistry on oral cancer screening. Oral Dis. 12(2), 194-99.

Lima, A.A.S. et al. (2005). Conhecimento de alunos universitários sobre câncer bucal. Rev Bras Cancerol. 51(4), 283-288.

Mendonça, E.F. (2005). Complicações bucais da quimioterapia e radioterapia no tratamento do câncer. Rev ABO Nac. 13(3), 151-7.

Morais, A. M. D. et al. (2017). Estudo das manifestações bucais de pacientes tratados com quimioterapia. Journal of orofacial investigation. 4(1). Faculdade de Ciência do Tocantins.

Morais, T. M. N. et al. (2006). A importância da atuação odontológica em pacientes internados em unidades de terapia intensiva. Rev Bras de Ter Intensiva. $18(4), 412-417$.

National Cancer Institute. (2018). Oral complications of cancer and cancer therapy. http:// cancerweb.ncl.ac.uk/cancernet/302904.html.

Organización Mundial De La Salud. (2014) Centro de prensa. Cáncer. 〈http://www.who.int/mediacentre/factsheets/fs297/es/index.html>.

Paiva, M. D. E. B., et al. (2010). Complicações orais decorrentes da terapia antineoplásica - Oral complications of cancer therapy. Arquivos em Odontologia. 46(1).

Pereira, J. V. et al. (2008). Avaliação de Streptococcus Mutans e velocidade do fluxo salivar em pacientes com câncer de cabeça e pescoço submetidos à quimioterapia e radioterapia. Pesq Bras Odontoped Clin Integr. 8(3), 295-9.

Pereira, P. L.; Nunes, A. L. S.\& Duarte, S. F. P. (2015). Qualidade de Vida e Consumo Alimentar de Pacientes Oncológicos. Revista Brasileira de Cancerologia. 3(61), 243-251.

Prado, B. N. \& PassarellI, D. H. C. (2009). Uma nova visão sobre prevenção do câncer bucal no consultório odontológico. Revista de Odontologia da Universidade Cidade de São Paulo. 21(1), 79-85.

Rosa, F. M.; Hammerschmitt, T. \& Souza, H. P. (2005) Utilização do laser de baixa potência na prevenção e terapêutica da mucosite oral. Stomatos. 11(21), 41-47.

Salazar, M. V. F. et al. (2008). Efeitos e tratamento da radioterapia de cabeça e pescoço de interesse ao cirurgião dentista. Revista Odonto. 16(31), 62-68.

Santos, C. C. S. et al. (2013). Condutas práticas e efetivas recomendadas ao cirurgião dentista no tratamento pré, trans e pós do câncer bucal. Curso de Odontologia da Universidade Paulista.

Santos, L. C. O.; Batista, O. M. \& Cangussu, M. C. T. (2010) Characterization of oral cancer diagnostic delay in the state of Alagoas. Braz J Otorhinolaryngol. 76(4), 416 - 422.

Sera, E.A.R. et al. (2013). Avaliação dos cuidados odontológicos pré e trans tratamento radioterápico. Braz J Periodontol. $23(3), 30$ - 38.

Silva, L.C.F. (2004). Avaliação dos fatores de risco dentais e de malignidade em radioterapia da cabeça e do pescoço. Rev. de Cirug. eTraum. Buco-maxilofacial. 3(4), 187-95.

Silva, J. L. F.\& Arruda, F. F. (2006). Radioterapia nos tumores de cabeça e pescoço: spectos gerais. In: Guimarães, J. R. Manual de Oncologia.

Simino, G. P. R.; Santos, C. B. \& Mishima, S. M. (2010). O Follow-up of Cancer Patients by Family Health Workers. Rev Latino-Am Enfermagem.

Tolentino, E. S. et al. (2011). Oral adverse effects of head and neck radiotherapy: care guideline for irradiated patients. J Appl Oral Sci. 5(19), 448-54.

Veit, M. T. \& Carvalho, V. A. (2010). Psico-Oncologia: um novo olhar para o câncer. O Mundo da Saúde. 34(4), 526-530.

Vissink, A. et al. (2003). Oral Sequelae of head and neck radiotherapy. Crit Rev Oral Biol Med. 14(3), 199-212.

Vieira, A. C. F. \& Lopes, F. F. (2006). Mucosite oral: efeito adverso da terapia antineoplásica. Revista de Ciências Médicas e Biológicas. 5(3), $268-274$.

Vilar, C. M. C. \& Martins, I. M. (2012). Princípios de cirurgia oncológica. In: Vieira, S. C. et al. Oncologia Básica. Teresina: Fundação Quixote. 